\title{
TRACKING FILTERS FOR RADAR SYSTEMS WITH CORRELATED MEASUREMENT NOISE
}

\author{
Donka ANGELOVA and Boryana VASSILEVA
}

\section{Introduction}

Most tracking filters are based on the Kalman filter equations, ${ }^{1}$ where the tracking system model presumes white process and measurement noise. In practice, the measurement noise may not be white. Its bandwidth may be on the order of several hertz. For example, target scintillation (or glint) causes the range and angle measurement errors to have a finite bandwidth. Another example of correlated measurement error is the radial velocity measurement error appearing as a result of radar frequency instability and target velocity fluctuations.

When the measurement frequency is much smaller than the error bandwidth, the errors of successive measurements are approximately uncorrelated and can be treated as white noise. However, the measurement frequency of some modern radars is sufficiently high and the correlation cannot be ignored without tracking accuracy deterioration.

A possible approach to circumvent the effect of colored noise is the target state augmentation technique. ${ }^{1}$ However, this yields a singular state covariance matrix, which may be ill-conditioned. A solution to the problem was suggested first by Bryson and Henrikson. ${ }^{2}$ They generated a pseudomeasurement, linear combination of two consecutive measurements, which is corrupted by white noise. In this measurement difference approach the application of the Kalman filter equations is straightforward. Rogers modeled colored noise as a first order Autoregressive (AR) process and applied the pseudomeasurement method to the $\alpha-\beta$ filter. ${ }^{3}$ But in realworld environment the exact prior information of the AR coefficients is not known. $\mathrm{Wu}$ and Chang proposed a method to estimate the AR parameters by removing the state variables from the measurements. ${ }^{4}$ Their method and the pseudo-measurement approach are used in the Interacting Multiple Model (IMM) filter for maneuvering target tracking. ${ }^{4}$ Thus, significant improvement is obtained. 
The application of the pseudomeasurement method is limited to the case where only position is measured. The case where both position and velocity are measured cannot be solved by this approach. Gazit ${ }^{5}$ extends the procedure suggested in [2] and formulates an optimal filter for tracking nonmaneuvering target without any restriction on the models' dimension. Using this decorrelation approach, a technique for tracking filters design for maneuvering targets is presented in this work. A new algorithm for AR parameter estimation is proposed. It is appropriate for on-line processing and is incorporated into the IMM filter. Two practical tasks are solved: 1) tracking with position measurements and 2) tracking with position and velocity measurements. The performances of the suggested algorithms are evaluated by Monte Carlo computer simulation.

The paper is organized as follows. Section 2 concisely summarizes the measurement decorrelation approach proposed in [5] and marks the IMM algorithm as an effective estimator of maneuvering targets. Section 3 presents a new algorithm for estimation of AR parameters based on the true state variable removing method. ${ }^{4}$ The Monte Carlo simulation results are described in section 4. Conclusions are summarized in the final section.

\section{Tracking filter design}

Tracking filters have to be correctly designed to obtain best results for a specific practical application. This process is often a trade-off among quality, complexity and possibility for on-line processing. The filter design comprises the choice of a measurement error model, the choice of a noise decorrelation scheme and a filtering algorithm, as well as the selection of their a priori parameters.

A. Measurement noise decorrelation. Consider the following model of a linear dynamic system for tracking with colored measurement noise ${ }^{5}$ :

$$
\begin{aligned}
& x(k+1)=\Phi x(k)+G w(k) \\
& e(k+1)=\Psi e(k)+\eta(k)
\end{aligned}
$$

where $x(k) \in \mathfrak{R}^{n_{x}}$ is the target state vector, $e(k) \in \mathfrak{R}^{n_{e}}$ is the state vector of the measurement error model, $w(k)$ and $\eta(k)$ are random noise sequences, assumed to be white and mutually uncorrelated with covariances $W(k)$ and $V(k)$ respectively. Let target position $x_{k}$, velocity $v_{k}$, and acceleration $a_{k}$ be components of the state vector in one-dimensional case: $x(k)=\left(x_{k}, v_{k}, a_{k}\right)^{T}$. The state transition matrix $\Phi$ and the noise matrix $G$ are determined by the target dynamics and assumed to be 
known to the tracking filter. The measurement vector $y(k) \in \mathfrak{R}^{n_{y}}$ in the measurement model

$$
y(k)=C x(k)+D e(k)
$$

is a linear combination of $x(k)$ and $e(k)$. The matrix $C$ selects the measured elements of $x(k)$ (for example, position only, or position and velocity). A state space partition and a reduced order dynamic model are used in [5] to construct a tracking filter without limitations concerning the order of the error model and measurement vector dimension. The author rewrites the measurement equation in the form:

$$
y(k)=x_{a}(k)+e_{a}(k)
$$

where $x_{a}(k) \in \mathfrak{R}^{n_{y}}$ and $e_{a}(k) \in \mathfrak{R}^{n_{y}}$ are the measured elements of $x(k)$ and $e(k)$. Thus a partition of the state vectors is actually formed. It imposes a similar partition of the transition matrices, noise vectors and their covariance matrices. Analogous to [2] a new measurement vector is defined:

$$
y_{r}(k)=y(k+1)-\Psi_{a} y(k)
$$

where $\Psi_{a}$ has dimension $\left(n_{y} \times n_{y}\right)$ and corresponds to the vector $e_{a}$. After appropriate substitutions and transformations the target and measurement equations are reformulated:

$$
\begin{aligned}
& x_{r}(k+1)=F x_{r}(k)+G_{r} u_{r}(k)+w_{r}(k) \\
& y_{r}(k)=H x_{r}(k)+\eta_{r}(k)
\end{aligned}
$$

where the deterministic input $u_{r}(k)=y(k)$ and the new state vector $x_{r}(k)$ of dimension $n_{r}=n_{x}+n_{e}-n_{y}$ is extended with the unmeasured elements of $e(k)$. The form of the system matrices $F, G_{r}, H$ can be found in [5]. The new process noise $w_{r}(k)$ and measurement noise $\eta_{r}(k)$ are now white sequences, but they are mutually correlated: $E\left[w_{r}(j) \eta_{r}(k)^{T}\right]=S \delta_{j k}$. Since the matrix $S$ is small (its elements contain the $6 \div 3$ degree of the sampling interval $T=0.05 s), w_{r}(k)$ can be assumed uncorrelated with $\eta_{r}(k)$ with slight degradation in performance. ${ }^{7}$ The system order $n_{r}=n_{x}+n_{e}-n_{y}$ is smaller than the order of the augmented system 
$n_{x}+n_{e} \cdot{ }^{1}$ Now the application of the Kalman filter becomes possible and, consequently, the IMM algorithm can be applied to the case of maneuvering target tracking with correlated measurement noise.

B. Measurement error model. The measurement error $e(k)$ is modeled as a first-order AR process. In that case $\Psi$ is the matrix of the AR parameters and $\eta(k)$ is a zero mean white Gaussian noise with variance $E\left[\eta(j) \eta(k)^{T}\right]=V \delta_{j k}$. When only the position of the target is measured, then the system parameters are determined as:

$$
n_{y}=1 ; n_{e}=1 ; x_{a}(k)=x_{k} ; \eta(k)=\eta_{k}^{x} ; \quad \Psi=\alpha^{x} ; \alpha^{x}=\exp (-\lambda T),
$$

where $\lambda$ is the bandwidth of the measurement noise. $\alpha_{x}$ and the noise variance $\left(\sigma_{k}^{\eta^{x}}\right)^{2}$ are two AR parameters which have to be estimated. When both position and velocity are measured, then:

$n_{y}=2 ; \quad n_{e}=2 ; \quad x_{a}(k)=\left(x_{k}, v_{k}\right)^{T} ; \eta(k)=\left(\eta_{k}^{x}, \eta_{k}^{v}\right)^{T} ; \Psi=\operatorname{diag}\left(\alpha^{x}, \alpha^{v}\right)$ and $\alpha^{x}, \alpha^{v},\left(\sigma_{k}^{\eta^{x}}\right)^{2},\left(\sigma_{k}^{\eta^{y}}\right)^{2}$ are subject to adaptive estimation.

C. IMM state estimation algorithm. ${ }^{1}$ The kinematic behavior of a maneuvering target can be suitably described in the terminology of the stochastic hybrid systems. The aim of the hybrid estimation is to assess the system state and behavior mode based on the sequence of the noisy measurements. Filtering algorithms in general consist of operating in parallel Kalman filters and Bayesian mechanism to organize the cooperation between the individual filters. An underlying Markov chain is assumed to govern the mode switching. The IMM algorithm is one of the most effective recent suboptimal Bayesian filters for hybrid system estimation. It provides the overall system state estimate $\hat{x}(k / k)=\sum_{j=1}^{r} \hat{x}^{j}(k / k) \mu_{j}(k)$ and estimates its associated covariance matrix $P(k / k)$ as a weighted sum of the estimates $\hat{x}^{j}(k / k)$ and its covariances $P^{j}(k / k)$, formed by $r$ mode-conditional parallel Kalman filters. The posterior mode probabilities $\mu_{j}(k)$ are calculated on the base of the likelihood of the measurement, received at the current time step.

D. IMM tracking filter design includes: a) selection of target motion models and their parameters; b) assignment of transition probabilities of the underlying Markov chain. The motion modes along one of the Cartesian coordinates are modeled by a second-order kinematic (nearly constant velocity) model for uniform motion and two third-order (nearly constant acceleration) models for the maneuvers. The process noise standard deviations are chosen after some simulation experiments as follows: $\sigma_{w_{r}}^{1}=10 \mathrm{~m} / \mathrm{s}^{2}$ for nonmaneuvering mode and $\sigma_{w_{r}}^{2}=90 \mathrm{~m} / \mathrm{s}^{2}$, $\sigma_{w_{r}}^{2}=150 \mathrm{~m} / \mathrm{s}^{2}$ respectively for the two maneuvering modes corresponding to 
different maneuver intensities. The Markovian transition probability matrix is chosen identical to [4] in order to compare the final results.

\section{Estimation of AR parameters}

A technique that can effectively estimate the AR parameters of the position and velocity measurement noises is proposed in this work. Since the measurement (3) contains state variables $x_{k}$ and $v_{k}$ the direct estimation of the $e_{k}^{x}$ and $e_{k}^{v}$ parameters $\left(e(k)=\left(e_{k}^{x}, e_{k}^{v}\right)^{T}\right)$ is difficult. It will be very helpful to remove state variables:

$$
x_{k}=x_{k-1}+v_{k-1} T+\frac{1}{2} a_{k-1} T^{2}, \quad v_{k}=v_{k-1}+a_{k-1} T,
$$

where $a_{k}$ is the acceleration of the target. Let $\xi_{k}$ denotes the true target position $\left(\xi_{k}=x_{k}\right)$ or velocity $\left(\xi_{k}=v_{k}\right)$. The following filtering operation is used to obtain a new signal $\bar{u}_{k}$ that does not involve $\xi_{k}$ :

$$
\begin{aligned}
& \bar{u}_{k}=\sum_{i=0}^{l} C_{l}^{i}(-1)^{i}\left(\xi_{k-i}+e_{k-i}^{\xi}\right)=\sum_{i=0}^{l} C_{l}^{i}(-1)^{i} \xi_{k-i}+\sum_{i=0}^{l} C_{l}^{i}(-1)^{i} e_{k-i}^{\xi}= \\
& =\sum_{i=0}^{l} C_{l}^{i}(-1)^{i} e_{k-i}^{\xi}+\frac{1}{l-1}\left(a_{k-1}-a_{k-l}\right) T^{l-1}
\end{aligned}
$$

where if $\xi_{k}=x_{k}$, then $l=3$ and if $\xi_{k}=v_{k}$, then $l=2$. Thus the z-transform of (7) is:

$$
\bar{u}(z)=\left(1-z^{-1}\right)^{l} e^{\xi}(z)+m(z), \quad m(z)=\frac{1}{l-1}\left(z^{-1}-z^{-l} a(z)\right) T^{l-1} .
$$

Note that $e_{k}^{\xi}$ is an AR process. According to (1) its transfer function is:

$$
e^{\xi}(z)=\frac{1}{1-\alpha^{\xi} z^{-1}} \eta^{\xi}(z)
$$

and $\bar{u}(z)$ is: 


$$
\bar{u}(z)=\frac{\left(1-z^{-1}\right)^{l}}{1-\alpha^{\xi} z^{-1}} \eta^{\xi}(z)+m(z)
$$

Passing $\bar{u}(z)$ through filter with transfer function

$$
F(z)=\frac{1}{\left(1-\rho z^{-1}\right)^{l}},
$$

where $0 \leq \rho \leq 1$, the output

$$
u(z)=\frac{\left(1-z^{-1}\right)^{l}}{1-\alpha^{\xi} z^{-1}\left(1-\rho z^{-1}\right)^{l}} \eta^{\xi}(z)+\frac{m(z)}{\left(1-\rho z^{-1}\right)^{l}}
$$

can be obtained. For nonmaneuvering $\left(a_{k-1}=a_{k-l}=0\right)$ and maneuvering with constant acceleration $\left(a_{k-1}-a_{k-l}=0\right)$ cases, the second term of the right-hand side of (12) is zero. If the value of $\rho$ is chosen to be one, $u_{k}$ is just the colored noise $e_{k}^{\xi}$, i.e.

$$
u_{k}=\alpha^{\xi} u_{k-1}+\eta_{k}^{\xi}
$$

Here an algorithm based on the Burg's method ${ }^{6}$ is proposed to estimate the AR parameters. Since $e_{k}^{\xi}$ is modeled as a real 1 st-order AR process, this algorithm has a simple recursive structure:

$$
\begin{aligned}
& a_{k}=\beta a_{k-1}+2 u_{k} u_{k-1}, \quad b_{k}=\beta b_{k-1}+u_{k}^{2}+u_{k-1}^{2}, \quad \alpha_{k}^{\xi}=\frac{a_{k}}{b_{k}} ; \\
& \left(\sigma_{k}^{e^{\xi}}\right)^{2}=\frac{1}{2(N-1)} b_{k} ; \quad\left(\sigma_{k}^{\eta^{\xi}}\right)^{2}=\left(1-\left(\alpha_{k}^{\xi}\right)^{2}\right)\left(\sigma_{k}^{e^{\xi}}\right)^{2}
\end{aligned}
$$

where $0<\beta<1$ is the forgetting factor and $N=1 /(1-\beta)$ is the effective memory. If $\beta$ is large, the algorithm convergence is slow and it cannot respond to the change of $\alpha^{\xi}$ quickly. Advantage of using large $\beta$ is the small estimation variance. On the contrary, small $\beta$ will let fast algorithm convergence. In this case, however, the estimation variance is large. A good compromise between convergence rate and estimation error is achieved for $\beta=0.99$. 
When the target acceleration is not constant $\left(a_{k-1}-a_{k-l} \neq 0\right)$ and $\rho=1$ the low frequency components of $m(z)$ in (12) will be greatly amplified and $u_{k}$ will be no longer equal to $e_{k}^{\xi}$. This problem can be overcome choosing $\rho<1$ and using the range of the real $e_{k}^{\xi}$ values. Thus, if $u_{k}^{2} \geq 9\left(\sigma_{k-1}^{e^{\xi}}\right)^{2}$ the previous scan estimates remain the same. In this way the parameter estimations are updated when

$$
u(z) \approx \frac{\left(1-z^{-1}\right)^{l}}{\left(1-\alpha^{\xi} z^{-1}\right)\left(1-\rho z^{-1}\right)^{l}} \eta^{\xi}(z) .
$$

It is clear from (15) that larger $\rho$ will give better results. However, too large values of $\rho$ will amplify the $m_{k}^{\xi}$. From (8), we find that $m_{k}^{\xi}$ is determined by the target acceleration difference $a_{k-1}-a_{k-l}$ and sampling period $T$. From experience it is found that the estimates are almost not affected for $\rho=0.97$ if $e_{k}^{\xi}=e_{k}^{x}$ and $\rho=0.90$ if $e_{k}^{\xi}=e_{k}^{v}$. But the estimates are then biased. The biases of the $e_{k}^{v}$ parameters estimates are significant and cannot be ignored. The unbiased estimates can be found using the following expressions ${ }^{4}$ :

$$
\begin{array}{r}
\alpha^{v}=\frac{-\left(\zeta_{3}+\zeta_{2}\right)}{2 \zeta_{3}}-\frac{\sqrt{\left(\zeta_{3}+\zeta_{2}\right)^{2}-4 \zeta_{3}\left(\zeta_{3}+\zeta_{2}+\zeta_{1}\right)}}{2 \zeta_{3}} \\
\left(\sigma^{\eta^{v}}\right)^{2}=\frac{-A+\left(\rho^{2}+\rho^{4}\right)\left(\bar{\alpha}^{v}\right)^{3}}{\left(1+\rho^{2}\right) \bar{\alpha}^{v}+(2 \rho-4)}\left(\bar{\sigma}^{e^{v}}\right)^{2}+ \\
+\frac{2 \rho\left(\rho^{2}-1\right)\left(\bar{\alpha}^{v}\right)^{2}-\left(\rho^{2}+1\right) \bar{\alpha}^{v}}{\left(1+\rho^{2}\right) \bar{\alpha}^{v}+(2 \rho-4)}\left(\bar{\sigma}^{e^{v}}\right)^{2},
\end{array}
$$

where

$$
\begin{aligned}
& \zeta_{3}=(\rho+1)^{3}, \zeta_{2}=\left(-2 \rho^{2}-10 \rho-4\right)+(-6 \rho-2) \bar{\alpha}^{v}, \\
& \zeta_{1}=\left(-\rho^{3}-3 \rho^{2}+5 \rho+7\right)+(8 \rho+8) \bar{\alpha}^{v}, \\
& A=-2 \rho^{3}\left(\bar{\alpha}^{v}\right)^{2}+\left(1-\rho^{4)} \bar{\alpha}^{v}+2 \rho\right.
\end{aligned}
$$


$\bar{\alpha}^{v}$ and $\left(\bar{\sigma}^{e^{v}}\right)^{2}$ are biased estimates denotations. The described AR parameter estimation algorithm has simple structure. Its complexity estimation includes 44 multiplications and divisions and one square root operation for one cycle if both position and velocity are measured. The computational complexity of this algorithm is approximately one-tenth of the IMM algorithm of target tracking.

\section{Computer simulation results}

The performance of the proposed algorithm is investigated by means of simulation analysis. The realized Monte Carlo simulation model implements the following tasks: simulates the real target dynamics; generates measurements according to the accepted noise model; implements the algorithm of interest; performs a posterior statistical processing of the experimental data.

The target motion scenario is chosen as follows. The maneuver lasts from 10 to $30 \mathrm{~s}$ with constant acceleration equal to $40 \mathrm{~m} / \mathrm{s}^{2}$ (about $4 \mathrm{~g}$ ). The sampling period $T$ is $0.05 s$. The total tracking interval is $50 s$ (1000 samples). It is assumed that the standard deviations of measurement noises are $\sigma^{\eta^{x}}=100 \mathrm{~m}$ and $\sigma^{\eta^{v}}=15 \mathrm{~m} / \mathrm{s}$. During the nonmaneuvering period $(1 \div 10 s ; 31 \div 50 s)$ the coefficients are: $\alpha^{x}=\exp (-4 T)=.8187 ; \alpha^{v}=\exp (-1 T)=.9512$. During the maneuvering period $(11 \div 30 \mathrm{~s}) \quad$ the coefficients are: $\quad \alpha^{x}=\exp (-10 T)=.6067$; $\alpha^{v}=\exp (-5 T)=.7788$. The tracker is initiated $20 s$ before the formal tracking period. The purpose is to investigate the steady state behavior of the algorithm. One hundred Monte Carlo runs are carried out and the average results are shown under the Root Mean Square Error (RMSE) criterion.

The estimation errors of the correlation coefficients $\left(\alpha^{x}, \alpha^{v}\right)$ and the noise standard deviations $\left(\sigma^{\eta^{x}}, \sigma^{\eta^{v}}\right)$ are indicated by the curves on figures 1 and 2 respectively. They show that in steady state the estimate errors of the parameters are quite small (less than $10 \%$ ). The results of the parameters estimation when only position is measured closely correspond to the ones of $\mathrm{Wu}$ and $\mathrm{Chang}^{4}$, but the computational complexity of their algorithm is approximately two times bigger. From the above results we know that the proposed algorithm can estimate the AR parameters effectively.

To achieve better tracking performance, we incorporate it into the IMM filter for maneuvering target tracking. The filters performance is examined over the described motion scenario in two cases: case 1 - position only measurements and case 2 - both position and velocity measurements. 


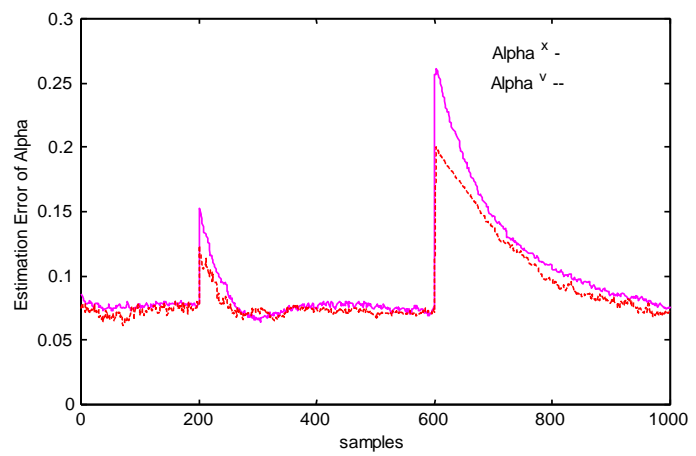

Figure 1

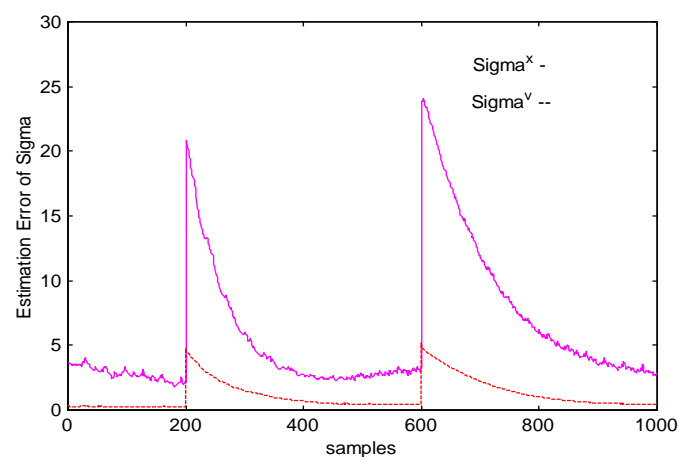

Figure 2

The filters efficiency is evaluated according to: RMS errors, Peak Dynamic Errors $(P D E)$, Correct Mode Identification (CMI). In figures 3 and 4 comparative velocity and acceleration RMS errors for tracking without decorrelation and with suggested adaptive decorrelation scheme are shown. From these figures we see that the noise decorrelation improves estimation accuracy, especially in the velocity and acceleration. In case 1 the improvement in velocity estimation is about $50 \%$ during uniform motion and $30 \%$ during maneuvering phase. For the acceleration these values are $60 \%$ and $30 \%$ respectively. The velocity measurement incorporation in case 2 additionally improves the estimation accuracy. In both cases, due to the measurement noises decorrelation, PDEs during maneuver on/off switching are considerably reduced. That can be seen from Table 1 as well. The evolution of the posterior probabilities corresponding to the three models of motion is presented on figure 5. It is seen that the IMM filter correctly identifies the true system mode (the delay in maneuver detection is about 20 sampling intervals). 


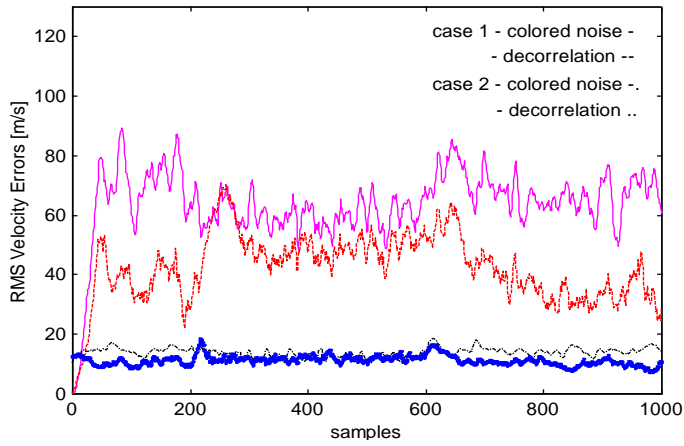

Figure 3



Figure 4

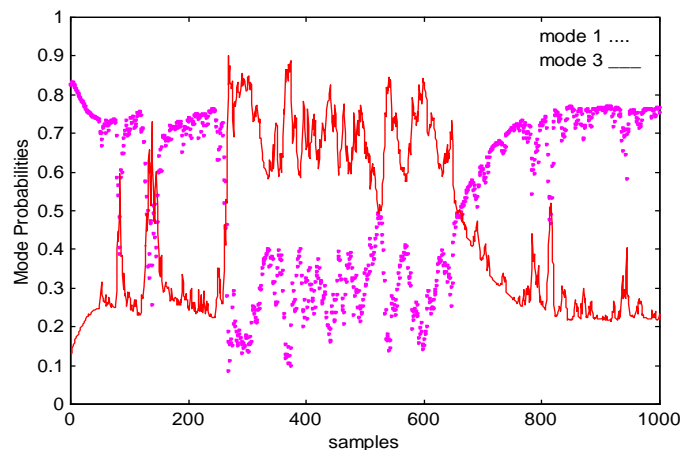

Figure 5 
Table 1

\begin{tabular}{|c|c|c|c|c|}
\hline Filter & Noise & $\begin{array}{c}\text { Position } \\
{[\mathrm{m}]}\end{array}$ & $\begin{array}{c}\text { Velocity } \\
{[\mathrm{m} / \mathrm{s}]}\end{array}$ & $\begin{array}{c}\text { Acceleration } \\
{\left[\mathrm{m} / \mathrm{s}^{2}\right]}\end{array}$ \\
\hline case 1 & undecorrelated & 89.23 & 85.33 & 51.85 \\
\hline & decorrelated & 76.92 & 64.00 & 40.74 \\
\hline case 2 & undecorrelated & 46.15 & 18.66 & 50.62 \\
\hline & decorrelated & 40.00 & 18.60 & 38.21 \\
\hline
\end{tabular}

\section{Conclusions}

Tracking filters for radar systems with correlated measurement noise are developed in this work. Two practical tasks are solved: 1) tracking with only position measurements and 2) tracking with position and velocity measurements. The noise decorrelation approach and state space partition are applied for tracking maneuvering objects with two-dimensional measurement vector. A new algorithm based on removing the state variables from measurements is proposed to identify the parameters of the colored noise. This decorrelation scheme is included into the costeffective IMM filter. Simulation results demonstrate fairly better tracking accuracy compared to the undecorrelated measurement errors and almost the same estimation capabilities as in the case of exactly decorrelated measurement errors. The filter structure is simple, practically feasible and suitable for on-line processing. In the measurement equation, only colored noise which is modeled as a first-order AR process is assumed. In real applications white noise also exists in the measurement errors and produces an Autoregressive Moving Average (ARMA) noise process. In this case, the described decorrelation algorithm becomes suboptimal.

\section{Acknowledgement}

This study is supported by the Bulgarian National Foundation for Scientific Investigations grant I-808/98. 


\section{References}

1. Y. Bar-Shalom and X. Rong-Li, Estimation and tracking: Principles, techniques and software (Boston, MA: Artech House, 1993).

2. A.E. Bryson and L.J. Henrikson, "Estimation using sampled data containing sequentially correlated noise," Journal of Spacecraft 5, 6 (1968), 662-665.

3. S.R. Rogers, "Alpha-beta filter with correlated measurement noise," IEEE, AES-23, 4 (1987), 592-594.

4. Wen-Rong $\mathrm{Wu}$ and Dah-Chung Chang, "Maneuvering target tracking with colored noise," IEEE AES-32, 4 (1996), 1311-1320.

5. Ran Gazit, "Digital tracking filters with high order correlated measurement noise," IEEE AES-33, 1 (1997), 171-177.

6. S.M. Kay and S.L. Marple, "Spectrum analysis. A modern perspective," IEEE 69, 11 (1981).

7. E. Mazor, A. Averbuch, Y. Bar-Shalom and J. Dayan, "Interacting multiple model methods in target tracking: a survey," IEEE AES-34, (1998), 103-123.

8. B. Ozkaya and C. Cengiz Arcasoy, "Analytical Solution of discrete colored noise ECA Tracking filter,” IEEE AES-34, 1 (1998), 93-102. 